\title{
Submatrices of Four Dimensional Summability Matrices
}

\author{
FATIH NURAY \\ Department of Mathematics, \\ AfyonKocatepe University, Afyonkarahisar, Turkey, \\ Deparment of Mathematics and Statistics, University of North Florida, Jacksonville, FL, USA \\ University of North Florida, Jacksonville, FL, USA \\ fnuray@aku.edu.tr \\ Richard F. PATTERSON \\ Department of Mathematics and Statistics, \\ University of North Florida Jacksonville, \\ Florida, 32224 \\ rpatters@unf.edu
}

\begin{abstract}
In this paper, we show that a matrix that maps $\ell^{\prime \prime}$ into $\ell^{\prime \prime}$ can be obtained from any $\mathrm{RH}$-regular matrix by the deletion of rows. Also a four dimensional conservative matrix can be obtained by the deletion of rows from a matrix that preserves boundedness. We will use these techniques to derive a sufficient condition for a four dimensional matrix to sum an unbounded sequence.

\section{RESUMEN}

En este trabajo probamos que una matriz que lleva $\ell^{\prime \prime}$ en $\ell^{\prime \prime}$ se puede obtener a partir de cualquier matriz RH-regular eliminando filas. También una matriz cuatro dimensional conservative se puede obteber eliminando filas en una matriz que preserva acotación. Usamos estas técnicas para encontrar una condición suficiente para que una matriz cuatro dimensional sume una sucesión no acotada.
\end{abstract}

Keywords and Phrases: Submatrix, four dimensional summability matrix, Double Sequences, Pringsheim Limit, RH-regular matrix

2010 AMS Mathematics Subject Classification: 40C05, 40D05. 


\section{Introduction}

The most well-known notion of convergence for double sequences is the convergence in the sense of Pringsheim. Recall that a double sequence $x=\left\{x_{k, l}\right\}$ of complex (or real) numbers is called convergent to a scalar $\ell$ in Pringsheim's sense (denoted by P-lim $x=\ell$ ) if for every $\epsilon>0$ there exists an $N \in \mathbb{N}$ such that $\left|x_{k, l}-\ell\right|<\epsilon$ whenever $k, l>N$. Such an $x$ is described more briefly as "P-convergent". It is easy to verify that $x=\left\{x_{k, l}\right\}$ convergences in Pringsheim's sense if and only if for every $\epsilon>0$ there exists an integer $N=N(\epsilon)$ such that $\left|x_{i, j}-x_{k, l}\right|<\epsilon$ whenever $\min \{i, j, k, l\} \geq N$. A double sequence $x=\left\{x_{k, l}\right\}$ is bounded if there exists a positive number $M$ such that $\left|x_{k, l}\right| \leq M$ for all $k$ and $l$, that is, if $\sup _{k, l}\left|x_{k, l}\right|<\infty$.

A double sequence $x=\left\{x_{k, l}\right\}$ is said to convergence regularly if it converges in Pringsheim's sense and, in addition, the following finite limits exist:

$$
\begin{aligned}
& \lim _{k \rightarrow \infty} x_{k, l}=\ell_{l} \quad(l=1,2, \ldots), \\
& \lim _{l \rightarrow \infty} x_{k, l}=\mathcal{L}_{k} \quad(k=1,2, \ldots) .
\end{aligned}
$$

Note that the main drawback of the Pringsheim's convergence is that a convergent sequence fails in general to be bounded. The notion of regular convergence lacks this disadvantage.

A double sequence $x$ is divergent in the Pringsheim sense(P-divergent) provided that $x$ does not convergence in the Pringsheim sense.

Let $A=\left(a_{m, n, k, l}\right)$ denote a four dimensional summability method that maps the complex double sequence $x$ into the double sequence $A x$ where the $m n$-th term to $A x$ is as follows:

$$
(A x)_{m, n}=\sum_{k=0}^{\infty} \sum_{l=0}^{\infty} a_{m, n, k, l} x_{k, l} .
$$

In [12] Robison presented the following notion of regularity for four-dimensional matrix transformation and a Silverman-Toeplitz type characterization of such notion.

Definition 1.1. The four-dimensional matrix $\mathrm{A}$ is said to be RH-regular if it maps every bounded $P$-convergent sequence into a P-convergent sequence with the same P-limit.

The assumption of bounded was added because a double sequence which is $\mathrm{P}$-convergent is not necessarily bounded.

The four-dimensional matrix $\mathrm{A}$ is said to be $\mathrm{RH}$-conservative if it maps every bounded $\mathrm{P}$ convergent sequence into a P-convergent sequence. In [12, Robison presented the following notion of a conservative four-dimensional matrix transformation and a SilvermanToeplitz type characterization of such a notion.

Theorem 1.2. ([5],[12]) The four-dimensional matrix $\mathrm{A}$ is RH-conservative if and only if

$$
\mathrm{RH}-\mathrm{C}_{1}: P-\lim _{\mathrm{m}, \mathrm{n}} \mathrm{a}_{\mathrm{m}, \mathrm{n}, \mathrm{k}, \mathrm{l}}=\mathrm{c}_{\mathrm{kl}} \text { for each } \mathrm{k} \text { and } \mathrm{l} \text {; }
$$




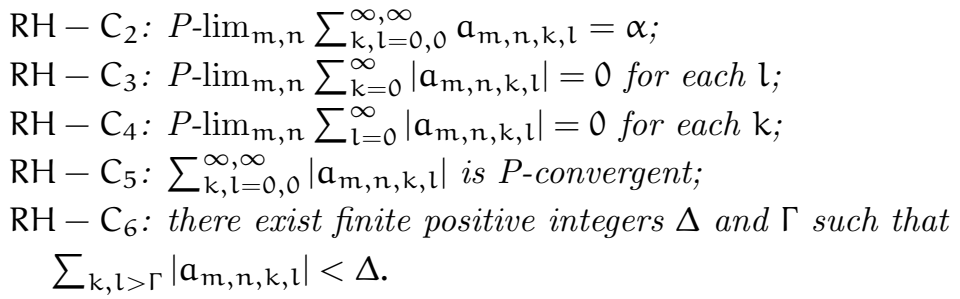

Along these same lines, Robison and Hamilton presented a Silverman-Toeplitz type multidimensional characterization of regularity in [5] and [12].

Theorem 1.3. (Hamilton [5], Robison [12]) The four-dimensional matrix $A$ is RH-regular if and only if

$\mathrm{RH}_{1}: P-\lim _{\mathrm{m}, \mathrm{n}} \mathrm{a}_{\mathrm{m}, \mathrm{n}, \mathrm{k}, \mathrm{l}}=0$ for each $\mathrm{k}$ and $\mathrm{l}$;

$\mathrm{RH}_{2}: P-\lim _{\mathrm{m}, \mathrm{n}} \sum_{\mathrm{k}, \mathrm{l}=0,0}^{\infty, \infty} \mathrm{a}_{\mathrm{m}, \mathrm{n}, \mathrm{k}, \mathrm{l}}=1$;

$\mathrm{RH}_{3}: P-\lim _{\mathrm{m}, \mathrm{n}} \sum_{\mathrm{k}=0}^{\infty}\left|\mathrm{a}_{\mathrm{m}, \mathrm{n}, \mathrm{k}, \mathrm{l}}\right|=0$ for each $\mathrm{l}$;

$\mathrm{RH}_{4}: P-\lim _{\mathrm{m}, \mathrm{n}} \sum_{\mathrm{l}=0}^{\infty}\left|\mathrm{a}_{\mathrm{m}, \mathrm{n}, \mathrm{k}, \mathrm{l}}\right|=0$ for each $\mathrm{k}$;

$\mathrm{RH}_{5}: \sum_{\mathrm{k}, \mathrm{l}=0,0}^{\infty, \infty}\left|\mathrm{a}_{\mathrm{m}, \mathrm{n}, \mathrm{k}, \mathrm{l}}\right|$ is $P$-convergent;

$\mathrm{RH}_{6}$ : there exist finite positive integers $\Delta$ and $\Gamma$ such that

$\sum_{k, l>\Gamma}\left|a_{m, n, k, l}\right|<\Delta$.

The set of all absolutely convergent double sequences will be denoted $\ell^{\prime \prime}$, that is

$$
\ell^{\prime \prime}=\left\{x=\left\{x_{k, l}\right\}: \quad \sum_{m=0}^{\infty} \sum_{n=0}^{\infty}\left|x_{k, l}\right|<\infty\right\} .
$$

In [9, Patterson proved that the matrix $A=\left(a_{m, n, k, l}\right)$ determines an $\ell^{\prime \prime}-\ell^{\prime \prime}$ method if and only if

$$
\sup _{k, l} \sum_{m=0}^{\infty} \sum_{n=0}^{\infty}\left|a_{m, n, k, l}\right|<\infty .
$$

In this paper, firstly we will obtain a general correspondance between RH-regular matrices and $\ell^{\prime \prime}-\ell^{\prime \prime}$ matrices by proving that every RH-regular matrix gives rise to an $\ell^{\prime \prime}-\ell^{\prime \prime}$ matrix by the removing of appropriate rows. Secondly we prove that a matrix from double bounded sequences to double bounded sequences contains a row-submatrix is conservative. Lastly, in order to prove a criterion for the summability of an unbounded double sequence the row-selection technique will be replaced by a column-selection technique.

\section{Main Results}

Theorem 2.1. If $\mathrm{A}=\left(\mathrm{a}_{\mathrm{mnkl}}\right)$ is a four dimensional summability matrix in which each row and each column converge to zero and

$$
\sup _{m, n}\left|a_{m n k l}\right|=\alpha<\infty
$$


for each $\mathrm{k}$ and $\mathrm{l}$ then $\mathrm{A}$ contains a row-submatrix that is an $\ell^{\prime \prime}-\ell^{\prime \prime}$ matrix.

Proof. First chose positive integer $v_{0}$ and $w_{0}$ satisfying $\left|a_{v_{0}, w_{0}, 0,0}\right| \leq 1$; then, using the assumption that

$$
P-\lim _{k, l} a_{v_{0}, w_{0}, 0,0}=0,
$$

choose $k_{0}$ and $l_{0}$ so that $k>k_{0}$ and $l>l_{0}$ implies $\left|a_{v_{0}, w_{0}, k, l}\right| \leq 1$. Having selected $v_{i}, w_{j}, k_{i}$ and $l_{j}$ for $i<p$ and $j<q$ we choose $v_{p}>v_{p-1}$ and $w_{q}>w_{q-1}$ so that

$$
k \leq k_{p-1}, \quad l \leq l_{q-1} \quad \text { implies } \quad\left|a_{v_{p}, w_{q}, k, l}\right| \leq 2^{-(p+q)}
$$

then choose $k_{p}>k_{p-1}$ and $l_{q}>l_{q-1}$ so that

$$
k>k_{p}, l>l_{q} \text { implies }\left|a_{v_{p}, w_{q}, k, l}\right| \leq 2^{-(p+q)} .
$$

Now define the submatrix $B$ by $b_{m n k l}=a_{v_{p}, w_{q}, k, l}$. The above construction guarantees that each column sequence of $B$ is dominated, except for at most one term, by the sequence $\left(2^{-(p+q)}\right)$; that is, if $k_{p-1}<k \leq k_{p}, l_{q-1}<l \leq l_{q}, i \neq p$ and $j \neq q$, then $\left|b_{i j k l}\right|=\left|a_{v_{i}, w_{j}, k, l}\right| \leq 2^{-(i+j)}$. Since $\left|a_{v_{p}, w_{q}, k, l}\right| \leq \alpha$, it is clear that for each $k, l$,

$$
\sum_{p=0}^{\infty} \sum_{q=0}^{\infty}\left|b_{p q k l}\right| \leq 4+\alpha .
$$

Hence, by (1.1), B is an $\ell^{\prime \prime}-\ell^{\prime \prime}$ matrix.

Corollary 2.2. Every four dimensional RH-regular matrix contains a row-submatrix that is an $\ell^{\prime \prime}-\ell^{\prime \prime}$ matrix.

Theorem 2.3. If A maps double bounded sequences into itself, then A contains a conservative row-submatrix $\mathrm{B}$.

Proof. Since A maps double bounded sequences into itself, we have

$$
\sup _{m, n} \sum_{k} \sum_{l}\left|a_{m n k l}\right|<\infty \text {. }
$$

Therefore the sequence of row sums $\left(\sum_{k} \sum_{l} a_{m n k l}\right)$ is bounded, so we can choose a convergent subsequence. This yields a row-submatrix $A^{\prime}$ of $A$ that satisfies properties (ii) and (iii). It remains to choose a row-submatrix of $A^{\prime}$ whose columns are convergent sequences. But this is a special case of the familiar diagonal process that is used in the proof of the multidimensional analog of Helly Selection Principle (see [13, 22])for we have a family of functions (the rows of $A^{\prime}$ ) that are 
uniformly bounded by $\sup _{m, n} \sum_{k} \sum_{l}\left|a_{m n k l}\right|$ on their countable domain

$$
\left\{\begin{array}{cccccc}
(0,0), & (0,1), & (0,2), & (0,3), & (0,4), & \ldots \\
(1,0), & (1,1), & (1,2,) & (1,3), & (1,4), & \ldots \\
(2,0,) & (2,1), & (2,2), & (2,3), & (2,4), & \ldots \\
\vdots & \vdots & \vdots & \vdots & \vdots & \ddots
\end{array}\right\}
$$

Therefore we can select a sequence of these functions that converges at each $k$ and $l$. This sequence of rows of $A^{\prime}$ are then the rows of $B$.

\section{Summability of Unbounded Sequences}

In [8] Patterson and Savaş proved multidimensional generalization of Agnew's theorem.

Theorem 3.1. If the four dimensional matrix $A=\left(a_{m n k l}\right)$ such that

$$
\sum_{k} \sum_{l}\left|a_{m n k l}\right|<\infty \text { and } P-\lim _{m, n \rightarrow \infty} \sup _{k, l}\left|a_{m n k l}\right|=0,
$$

then there exists at least one P-divergent double sequence of zeros and ones that is A summable.

By modifying the proof of Theorem 2.1 from row selection to column selection, we can prove a theorem in which we relax the regularity of $A$, weaken property (3.1), and construct an unbounded sequence that is summed by $A$.

Theorem 3.2. If $\mathrm{A}$ is a four dimensional summability matrix whose column sequences tend to zero and

$$
\lim \inf _{k, l}\left\{\max _{\mathfrak{m}, \mathfrak{n}} \mid \mathbf{a}_{\mathbf{m n k l}}\right\} \mid=0,
$$

then $\mathrm{A}$ sums an unbounded sequence.

Proof. Using (3.2), we choose increasing sequences of column indices $\left(k_{p}\right)$ and $\left(l_{q}\right)$ such that for each $p$ and $q$,

$$
\max _{m, n}\left|a_{m n k_{p}} l_{q}\right|<2^{-(p+q)} .
$$

Then choose increasing row indices $\left(v_{p}\right)$ and $\left(w_{q}\right)$ so that if $k \leq k_{p}, l \leq l_{q}, m>v_{p}$ and $n>w_{q}$, then $\left|a_{m n k l}\right|<2^{-(p+q)}$. Now define double sequence $x$ by

$$
x_{k l}=\left\{\begin{array}{cc}
(p+1)(q+1), & \text { if } k=k_{p}, l=l_{q} \text { for some } p, q \\
0, & \text { otherwise }
\end{array}\right.
$$


Then $\mathrm{m}>v_{\mathrm{p}}$ and $\mathrm{n}>\boldsymbol{w}_{\mathrm{q}}$ implies

$$
\begin{aligned}
\left|(A x)_{m, n}\right| & =\left|\sum_{i=0}^{\infty} \sum_{j=0}^{\infty} a_{m n k_{i} l_{j}} x_{k_{i} l_{j}}\right| \\
& \leq \sum_{i=0}^{p} \sum_{j=0}^{q}(i+1)(j+1) 2^{-(p+q)}+\sum_{i=p+1}^{\infty} \sum_{j=q+1}^{\infty}(i+1)(j+1) 2^{-(i+j)} \\
& =\left((p+1)(p+2)(q+1)(q+2) 2^{-(p+q)-2}+\mathcal{R}_{p q},\right.
\end{aligned}
$$

where $\lim _{p, q} \mathcal{R}_{p q}=0$. Hence, $\lim _{m, n}(A x)_{m, n}=0$.

We note that if the row sequences of $A$ tend to zero then (3.1) implies

$$
\lim _{k, l \rightarrow \infty}\left\{\max _{m, n} \mid a_{m n k l}\right\} \mid=0,
$$

which stronger than (3.2). Therefore Theorem 3.2 does have a weaker hypothesis than Theorem 3.1 .

\section{Received: January 2014. Accepted: January 2015.}

\section{References}

[1] R. P. Agnew Inclusion relations among methods of summability compounded form given matrix methods, Ark. Mat. 2, (1952), 361-374.

[2] D. Djurcič, L. D. R. Kočinac, and M. R. Žižović, Double Sequences and Selections, Abstract and Applied Analysis, Volume 2012, Article ID 497594, 6 pages .

[3] J. A. Fridy, Absolute summability matrices that are stronger than the identity mapping, Proc. Amer. Math. Soc. 47, (195), 112-118.

[4] J. A. Fridy, Submatrices of summability matrices, Internat. J. Math. and Math. Sci., 1, (1978,) $519-524$.

[5] H. J. Hamilton, Transformations of multiple sequences, Duke Math. Jour., 2 (1936), 29 - 60.

[6] G. H. Hardy, Divergent series, Oxford, (1949).

[7] R. F. Patterson and E. Savaş, Matrix summability of statistically P-convergence sequences, Filomat 25, (4), (2011), 55-62.

[8] R. F. Patterson, A theorem on entire four dimensional summability methods, Appl. Math. Comput., 219, (2013), 7777-7782. 
[9] R. F. Patterson, Four dimensional matrix characterization of absolute summability, Soochow Journal of Math., 30 (1), (2004), 21-26.

[10] R. F. Patterson, Analogues of some fundamental theorems of summability theory, Internat. J. Math. \& Math. Sci. 23 (1), (2000), 1-9.

[11] A. Pringsheim, Zur theorie der zweifach unendlichen zahlenfolgen, Mathematische Annalen, 53, (1900), 289- 32 .

[12] G. M. Robison, Divergent double sequences and series, Amer. Math. Soc. Trans. 28, (1926), 50-73.

[13] V. C. Vyacheslav and M. Caterina A pointwise selection principle for metric semigroup valued functions, J. Math. Anal. Appl. 341, (2008), 613-625. 